\title{
Eigenfunctions of the Koch Snowflake Domain
}

\author{
Michel L. Lapidus ${ }^{1, \star}$, Michael M.H. Pang ${ }^{2, \star \star}$ \\ 1 Department of Mathematics, Sproul Hall, The University of California, Riverside, CA 92521- \\ 0135, USA,E-mail: Lapidus@math.ucr.edu \\ 2 Department of Mathematics, White Hall, Cornell University, Ithaca, NY 14853, USA, E-mail: \\ Pang@math.cornell.edu
}

Received: 5 May 1994

\begin{abstract}
We obtain non-tangential boundary estimates for the Dirichlet eigenfunctions $\varphi_{n}$ and their gradients $\left|\nabla \varphi_{n}\right|$ for a class of planar domains $\Omega$ with fractal boundaries. This class includes the quasidiscs and, in particular, snowflake-type domains. When applied to the case when $\Omega$ is the Koch snowflake domain, one of our main results states that $\left|\nabla \varphi_{1}(\omega)\right|$ tends to $\infty$ or 0 as $\omega$ approaches certain types of boundary points (where $\varphi_{1}>0$ denotes the ground state eigenfunction of the Dirichlet Laplacian on $\Omega$ ). More precisely, let $\mathrm{Ob}$ (resp., Ac) denote the set of boundary points which are vertices of obtuse (resp., acute) angles in an inner polygonal approximation of the snowflake curve $\partial \Omega$. Then given $v \in \mathrm{Ob}$ (resp., $v \in$ Ac), we show that $\left|\nabla \varphi_{1}(\omega)\right| \rightarrow \infty$ (resp., 0 ) as $\omega$ tends to $v$ in $\Omega$ within a cone based at $v$. Moreover, we show that blowup of $\left|\nabla \varphi_{1}\right|$ also occurs at all boundary points in a Cantor-type set. These results have physical relevance to the damping of waves by fractal coastlines, as pointed out by Sapoval et al. in their experiments on the "Koch drum".
\end{abstract}

\section{Introduction}

Let $\Omega$ be a bounded simply connected John domain in $\mathbb{R}^{2}$ and assume that the boundary $\partial \Omega$ of $\Omega$ is a Jordan curve. Let $-\Delta_{\Omega} \geqq 0$ be the Dirichlet Laplacian defined in $L^{2}(\Omega)$ and let $0<E_{1}<E_{2} \leqq \cdots$ be the eigenvalues of $-\Delta_{\Omega}$. Let $\varphi_{n}$ be the eigenfunction corresponding to $E_{n}$ and normalized by $\left\|\varphi_{n}\right\|_{2}=1$ (we assume that $\varphi_{1}>0$ ). In Sect. 2 (Theorem 2.8) we obtain an upper bound for $\varphi_{1}(x)$ as $x$ approaches a boundary point $v \in \partial \Omega$ while remaining inside a "twisting cone" with vertex at $v$. This upper bound is expressed in terms of the "average angle" of $\partial \Omega$ at $v$. If $\Omega$ is a polygon in $\mathbb{R}^{2}$ and $v$ is a corner of $\partial \Omega$, then our upper bound reduces to the usual form

$$
\varphi_{1}(x) \leqq c \operatorname{dist}(x, \partial \Omega)^{\pi / \theta},
$$

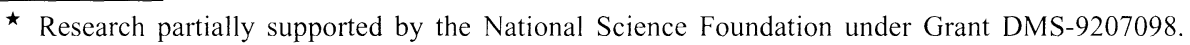
$\star \star$ Current address: Department of Mathematics, University of Missouri, Columbia, MO 65211, USA.
} 
where $\theta$ is the angle of $\partial \Omega$ at $v$ and $c \geqq 1$ is a constant depending only on $\Omega$. In Theorem 2.11 we establish an upper bound for $\left|\nabla \varphi_{n}(x)\right|$, the magnitude of the gradient of $\varphi_{n}$, of the form

$$
\left|\nabla \varphi_{n}(x)\right| \leqq k_{n} \varphi_{1}(x) \operatorname{dist}(x, \partial \Omega)^{-1}
$$

as $x$ approaches a point $v \in \partial \Omega$ while remaining inside a "twisting cone" $U$ with vertex at $v$. For $\varphi_{1}$ and for domains such as the snowflake region, we also prove a lower bound of the same form

$$
\left|\nabla \varphi_{1}(x)\right| \geqq k \varphi_{1}(x) \operatorname{dist}(x, \partial \Omega)^{-1} \quad(x \in U) .
$$

In Sect. 3 we apply our results in Sect. 2 to the case when $\Omega$ is the (Koch) snowflake domain. Our main result in this section is Theorem 3.2. Let $O b$ (resp., $A c$ ) be the set of boundary points which are vertices of obtuse (resp., acute) angles in an inner polygonal approximation of the snowflake. Then given $v \in O b$ (resp., $v \in A c$ ), we show that $\left|\nabla \varphi_{1}(\omega)\right| \rightarrow \infty$ (resp., $\rightarrow 0$ ) as $\omega$ tends to $v$ in $\Omega$ within a cone based at $v$. Moreover we also show that blowup of $\left|\nabla \varphi_{1}\right|$ occurs at all boundary points in a Cantor-type set. The potential significance of the blowup of $\left|\nabla \varphi_{n}\right|$ (for the damping of waves by fractal coastlines, for example) was pointed out by $B$. Sapoval and his collaborators $[\mathrm{S}, \mathrm{SGM}, \mathrm{SG}]$ in the appealing interpretation of their physical experiments on the vibrations of the "Koch drum."

For other aspects of the spectral theory of "drums with fractal boundary," we refer the reader, e.g., to the survey article [L].

For the rest of this paper a bounded simply connected region $\Omega$ in $\mathbb{R}^{2}$ is a John domain if there exists $M \geqq 1$ such that for every rectilinear crosscut $[a, b]$ of $\Omega$,

$$
\operatorname{diam}(H) \leqq M|a-b|
$$

holds for one of the two components $H$ of $\Omega \backslash[a, b]$ (see [P, p. 96]). It can be shown that all quasidiscs, in particular the snowflake domain, are John domains (see [P, p. 107]).

\section{Bounded Simply Connected John Domains}

In this section we let $\Omega \subseteq \mathbb{R}^{2}$ be a bounded simply connected John domain whose boundary $\partial \Omega$ is a Jordan curve. We let $v$ be an arbitrary but fixed point on $\partial \Omega$. " $c$ " will denote constants $(\geqq 1)$ which depend only on $\Omega$ and $v$. We shall write $-\Delta_{\Omega}$ for the Dirichlet Laplacian in $L^{2}(\Omega)$ and write $0<E_{1}<E_{2} \leqq E_{3} \leqq \cdots$ for the eigenvalues of $-\Delta_{\Omega}$ counted according to multiplicities. We let $\varphi_{n}$ be the eigenfunction corresponding to $E_{n}$ and normalized by $\left\|\varphi_{n}\right\|_{2}=1$ (we assume that $\varphi_{1}>0$ ). We shall use " $G_{\Omega}(x, y)$ " to denote the integral kernel of $\left(-\Delta_{\Omega}\right)^{-1}$ and write

$$
d(x)=d_{\Omega}(x)=\operatorname{dist}(x, \partial \Omega) \quad(x \in \Omega) .
$$

" $D$ " will denote the unit disk in $\mathbb{R}^{2}$. We first state the two main results from [DS, $\mathrm{RW}$ ] on which the proofs in this section are based. For the boundary behavior of conformal maps defined on $D$ we refer the readers to the excellent book $[\mathrm{P}]$ by Pommerenke.

Definition 2.1. We say that $e^{\Delta t}$ is intrinsically ultracontractive if the semigroup $\varphi_{1}^{-1} e^{\Delta t} \varphi_{1}$ on $L^{2}\left(\Omega, \varphi_{1}(x)^{2} d x\right)$ maps $L^{1}\left(\Omega, \varphi_{1}(x)^{2} d x\right)$ to $L^{\infty}\left(\Omega, \varphi_{1}(x)^{2} d x\right)$ as a 
bounded operator; i.e.,

$$
\left\|\varphi_{1}^{-1} e^{\Delta t} \varphi_{1}\right\|_{L^{1}\left(\Omega, \varphi_{1}(x)^{2} d x\right) \rightarrow L^{\infty}\left(\Omega, \varphi_{1}(x)^{2} d x\right)} \leqq \tilde{c}(t)<\infty
$$

for all $t>0$.

Lemma 2.2. ([DS, p. 374], see also [D, p. 131]) Let $X$ be a bounded region in $\mathbb{R}^{N}$ and let $-\Delta_{X}$ be the Dirichlet Laplacian on X. Let $0<E_{1}<E_{2} \leqq \cdots$ be the eigenvalues of $-\Delta_{X}$ and let $\varphi_{n}\left(\varphi_{1}>0\right)$ be the corresponding eigenfunctions normalized by $\left\|\varphi_{n}\right\|_{2}=1$. Suppose the following conditions hold:

(i) $e^{\Delta t}$ is intrinsically ultracontractive,

(ii) $-\Delta_{X} \geqq k$ dist $(x, \partial X)^{-x}$ in the quadratic form sense for some $k, \alpha>0$.

(iii) there exist $c \geqq 1$ and $\beta>0$ such that

$$
\varphi_{1}(x) \geqq c^{-1} \operatorname{dist}(x, \partial X)^{\beta} \quad(x \in X) .
$$

Then for any fixed $y \in X$, there exist $k_{1} \geqq 1$ and $\varepsilon>0$ such that

$$
k_{1}^{-1} \varphi_{1}(x) \leqq G_{X}(x, y) \leqq k_{1} \varphi_{1}(x)
$$

for all $x \in X$ with $\operatorname{dist}(x, \partial X)<\varepsilon$. Also if

$$
\left\|\varphi_{1}^{-1} e^{\Delta t} \varphi_{1}\right\|_{L^{1}\left(X, \varphi_{1}(x)^{2} d x\right) \rightarrow L^{\infty}\left(X, \varphi_{1}(x)^{2} d x\right)} \leqq \tilde{c}(t)<\infty
$$

for all $t>0$, then

$$
\left|\varphi_{n}(x)\right| \leqq \tau_{n} \varphi_{1}(x) \quad(x \in X)
$$

where

$$
\tau_{n}=\inf \left\{\tilde{c}(t) e^{\left(E_{n}-E_{1}\right) t}: t>0\right\} .
$$

Lemma 2.3. ([RW], see also [P, p. 264]) Let $X$ be a bounded simply connected region in $\mathbb{C}$ and let $g: X \rightarrow D$ be a Riemann map which can be extended to a homeomorphism between $\bar{X}$ and $\bar{D}$. Let $v \in \partial X$ and let $\zeta=g(v)$. Let $\{T(\rho)\}_{0<\rho<\rho_{0}}$ be a family of circular crosscuts in $X$ converging to $v$. Let $B=\bigcup_{0<\rho<\rho_{0}} T(\rho)$ and for $\rho \in\left(0, \rho_{0}\right)$, let

$$
\lambda(\rho)=\text { length of } T(\rho) \text {. }
$$

Then

$$
\lim _{\substack{(\omega) \rightarrow b \\(\omega \in B}}|g(\omega)-\zeta| \exp \left\{\int_{\substack{0 \\|\omega-1|}}^{\rho_{0}} \frac{\pi}{\lambda(\rho)} d \rho\right\}<\infty
$$

exists (see Fig. 1 (a)). Moreover if

$$
X=\left\{v+\rho e^{i()}: 0<\rho<\rho_{0}, \theta_{-}(\rho)<\theta<\theta_{+}(\rho)\right\},
$$

where the functions $\theta_{ \pm}$are locally absolutely continuous and satisfy

$$
\int_{0}^{\rho_{0}}\left[\theta_{+}(\rho)-\theta_{-}(\rho)\right]^{-1}\left[\theta_{+}^{\prime}(\rho)^{2}+\theta_{-}^{\prime}(\rho)^{2}\right] \rho d \rho<\infty,
$$

then the limit in (2.1) is positive (see Fig. 1 (b)). 

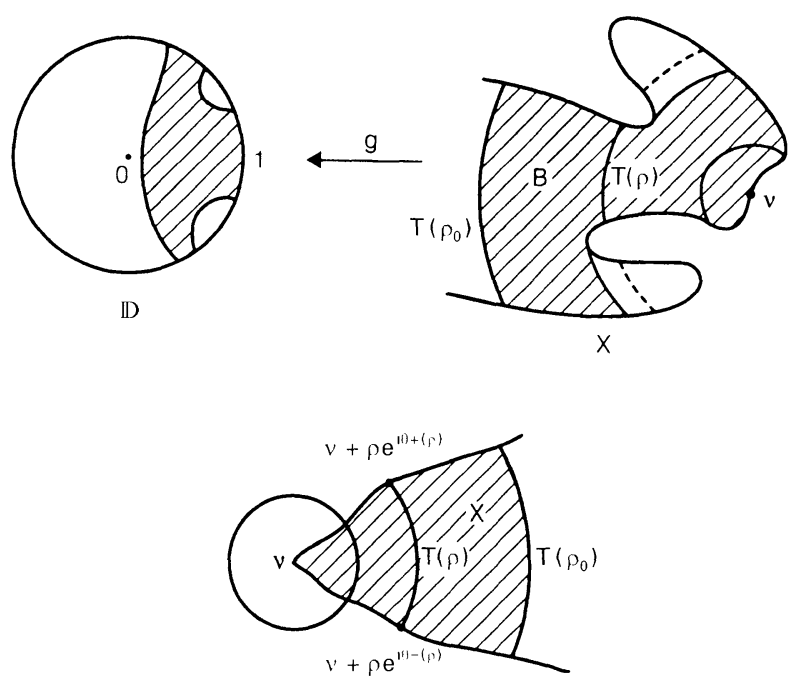

Fig. 1. The notation of Lemma 2.3

Definition 2.4. Let $v \in \partial \Omega$. A subset $U \subseteq \Omega$ is called a twisting cone in $\Omega$ with vertex at $v$ if it satisfies the following conditions: there exists a family of circular crosscuts $\{T(\rho)\}_{0<\rho \leqq \rho_{0}}$ converging to v such that

(TC1) $U$ is simply connected and $U^{\circ} \subseteq \Omega$.

(TC2) $\partial U$ consists of two curves $\gamma_{i}:[\overline{0}, 1] \rightarrow \bar{\Omega}, i=1$, 2, such that

(a) $\gamma_{1}([0,1)) \subseteq \Omega$ for $i=1,2$,

(b) $\left.\gamma_{1}([0,1)) \cap \gamma_{2}([0,1])\right)=\phi$,

(c) $\gamma_{1}(1)=\gamma_{2}(1)=v$.

(TC3) $U \cap T(\rho)$ is connected for all $\rho \in\left(0, \rho_{0}\right)$.

(TC4) There exists $c \geqq 1$ such that

$$
c^{-1} \lambda(T(\rho) \cap U) \leqq \min \left\{d\left(T(\rho) \cap \gamma_{l}([0,1))\right), i=1,2\right\}
$$

for all $\rho \in\left(0, \rho_{0}\right)$, where $\lambda(T(\rho) \cap U)$ is the length of $T(\rho) \cap U$ and $d(T(\rho) \cap$ $\left.\gamma_{1}([0,1))\right)$ is the distance of $T(\rho) \cap \gamma_{1}([0,1))$ from $\partial \Omega$.

(TC5) There exists a sequence $\rho_{1}>\rho_{2}>\rho_{3} \cdots \rightarrow 0$ and $c \geqq 1$ such that

(a) $\rho_{m} / \rho_{m+1} \leqq 2$ for $m=1,2,3, \ldots$;

(b) the distance between $T\left(\rho_{m}\right) \cap U$ and $T\left(\rho_{m+1}\right) \cap U$ in $U$ is at most $c \min \left\{d\left(T\left(\rho_{m}\right) \cap \gamma_{l}([0,1)), i=1,2\right\}\right.$.

(TC6) There exists $c \geqq 1$ such that

$$
0<c^{-1} \rho \leqq \min \left\{d\left(T(\rho) \cap \gamma_{i}([0,1))\right), i=1,2\right\} .
$$

Remarks. (i) In this paper we shall only use twisting cones of the type described in Proposition 2.7 below.

(ii) Suppose $U$ is a twisting cone with vertex at $v$. Assume that for every $m=$ $1,2,3 \ldots$ there exists $\omega_{m} \in T\left(\rho_{m}\right) \cap U$ such that

$$
\varphi_{1}\left(\omega_{m}\right) \leqq F\left(\rho_{m}\right)
$$


where $F$ is some explicit function. Then (TC4) $\rightarrow$ (TC6) together with the elliptic Harnack inequality imply that there exists $c \geqq 1$ such that

$$
\varphi_{1}(\omega) \leqq c F\left(\rho_{m}\right) \quad\left(\omega \in U \text { and } \rho_{m-1}>|\omega-v| \geqq \rho_{m}\right) .
$$

In particular if $F$ is of the form $F(\rho)=k \rho^{\gamma}$, for some $k, \gamma>0$, then we have

$$
\varphi_{1}(\omega) \leqq c|\omega-v|^{\prime} \quad(\omega \in U) .
$$

We next want to define a canonical twisting cone in $\Omega$ with vertex at $v$. To do this we shall need the following results:

Lemma 2.5. ([P, p. 97]) Let $X$ be a bounded simply connected region in $\mathbb{C}$ and let $f: D \rightarrow X$ be a Riemann map. For $r \in(0,1]$ and $t \in \mathbb{R}$, let

$$
B\left(r e^{i t}\right)=\left\{\rho e^{l \theta}: r \leqq \rho<1,|\theta-t| \leqq \pi(1-r)\right\} .
$$

Then the following are equivalent:

(i) $X$ is a John domain.

(ii) There exists $c_{1} \geqq 1$ such that

$$
\operatorname{diam}(f(B(z))) \leqq c_{1} d_{X}(f(z)) \quad(z \in D) .
$$

(iii) There exist $c \geqq 1$ and $\alpha \in(0,1]$ such that

$$
\left|f^{\prime}(\sigma)\right| \leqq c\left|f^{\prime}(z)\right|[(1-|\sigma|) /(1-|z|)]^{\alpha-1}
$$

for all $\sigma \in D \cap B(z)$ and $z \in D$.

Remark. If $\Omega$ is a bounded simply connected domain, then Lemma 2.5 (iii) implies that there exist $c_{2} \geqq 1$ and a point $\omega_{*} \in \Omega$ such that for any $\omega_{1} \in \Omega$ there is a rectifiable path $\gamma$ in $\Omega$ joining $\omega_{*}$ to $\omega_{1}$ such that

$$
l\left(\gamma\left(\omega, \omega_{1}\right)\right) \leqq c_{2} d(\omega) \quad(\omega \in \gamma),
$$

where $\gamma\left(\omega, \omega_{1}\right)$ is the part of $\gamma$ joining $\omega$ to $\omega_{1}$ and $l\left(\gamma\left(\omega, \omega_{1}\right)\right)$ is its arc length.

(Proof: Given $\omega_{1} \in \Omega$ we can assume that $f^{-1}\left(\omega_{1}\right) \in[0,1)$. Let $\omega_{*}=f(0)$ and let $\gamma(t)=f(t)$ for all $t \in[0,1)$. Then for any $\omega=\gamma(t), t \in\left[0, f^{-1}\left(\omega_{1}\right)\right]$

$$
\begin{aligned}
l\left(\gamma\left(\omega, \omega_{1}\right)\right) & \leqq \int_{t}^{1}\left|\gamma^{\prime}(u)\right| d u=\int_{t}^{1}\left|f^{\prime}(u)\right| d u \\
& \leqq c\left|f^{\prime}(t)\right|(1-t)^{1-\alpha} \int_{t}^{1}(1-u)^{\alpha-1} d u \\
& =c \alpha^{-1}\left|f^{\prime}(t)\right|(1-t) .
\end{aligned}
$$

Equation (2.4) together with Lemma 2.6 below now give (2.3).)

Lemma 2.6. (Koebe, see [P, p. 9]) If $f: D \rightarrow \mathbb{C}$ is an injective conformal map, then for all $z \in D$ we have

$$
\frac{\left|f^{\prime}(0)\right|(1-|z|)}{(1+|z|)^{3}} \leqq\left|f^{\prime}(z)\right| \leqq \frac{\left|f^{\prime}(0)\right|(1+|z|)}{(1-|z|)^{3}}
$$


and

$$
4^{-1}\left(1-|z|^{2}\right)\left|f^{\prime}(z)\right| \leqq \operatorname{dist}(f(z), \partial f(D)) \leqq\left(1-|z|^{2}\right)\left|f^{\prime}(z)\right|,
$$

where $\partial f(D)$ is the boundary of $f(D)$.

For all sufficiently small $\varepsilon, \rho_{0}>0$ we shall write

$$
Q\left(\varepsilon, \rho_{0}\right)=\left\{\omega \in \Omega:|\omega-v|<\rho_{0} \text { and } d(\omega)>\varepsilon|\omega-v|\right\} .
$$

For the rest of this paper we let $g: \Omega \rightarrow D$ be a fixed Riemann map with $g(v)=1$ and put $f=g^{-1}$.

Proposition 2.7. Let $c_{1}, c_{2} \geqq 1$ be chosen so that (2.2) and (2.3) hold. Then there exists $R>0$ such that if $0<\varepsilon \leqq\left(2 c_{1} c_{2}\right)^{-1}$, if $0<\rho_{0} \leqq R$ and if $\{V(\rho)\}_{0<\rho<\rho_{0}}$ is a family of circular crosscuts in $Q\left(\varepsilon, \rho_{0}\right)$ converging to $v$, then the set $U=$ $\bigcup_{0<\rho<\rho_{0}} V(\rho)$ is a twisting cone in $\Omega$ with vertex at $v$.

Proof. We first note that (2.3) implies that there exists a sufficiently small $R>0$ such that for any $\varepsilon \in\left(0,\left(2 c_{1} c_{2}\right)^{-1}\right]$ the part of the boundary of $Q(\varepsilon, R)$ inside $\Omega$ consists of two branches, one on each side of $v$. Hence if $0<\rho_{0} \leqq R$ and if $\{V(\rho)\}_{0<\rho<\rho_{0}}$ is a family of circular crosscuts in $Q\left(\varepsilon, \rho_{0}\right)$ converging to $v$, then $\partial U$ (where $U=\bigcup_{0<\rho<\rho_{0}} V(\rho)$ ) consists of two curves $\gamma_{l}:[0,1] \rightarrow \bar{\Omega}, i=1,2$, that satisfy the conditions in (TC2). Now $U$ clearly satisfies (TC1) and (TC3). (TC6) is true by the definitions of $Q\left(\varepsilon, \rho_{0}\right)$ and $U$. (TC4) is a consequence of (TC6). Finally we note that if $\varepsilon \in\left(0,\left(2 c_{1} c_{2}\right)^{-1}\right]$, then (2.2) implies that there exists $t_{0} \in(0,1)$ such that $f\left(\left(t_{0}, 1\right)\right) \subseteq Q\left(\varepsilon, \rho_{0}\right)$. Thus for every $\rho \in\left(0, \rho_{0}\right)$, there exists $\omega(\rho) \in f((0,1))$. Given a sequence $\rho_{0}>\rho_{1}>\rho_{2}>\cdots \rightarrow 0$ with $\rho_{m} / \rho_{m+1} \leqq 2$, the distance $d_{m}$ between $V\left(\rho_{m}\right)$ and $V\left(\rho_{m+1}\right)$ in $U$ satisfies

$$
\begin{aligned}
d_{m} & \leqq c\left|f^{\prime}\left(g\left(\omega\left(\rho_{m}\right)\right)\right)\right|\left(1-g\left(\omega\left(\rho_{m}\right)\right)\right) \\
& \leqq c d\left(\omega\left(\rho_{m}\right)\right) \\
& \leqq c \rho_{m} \\
& =c \varepsilon^{-1} \operatorname{dist}\left(V\left(\rho_{m}\right), \partial \Omega\right)
\end{aligned}
$$

where the first inequality of (2.7) follows from (2.4) and the second inequality from (2.6). (TC5) now follows from (2.7).

For the rest of this paper we shall denote by $U$ a fixed twisting cone in $\Omega$ with vertex at $v$ of the type described in Proposition 2.7.

Theorem 2.8. Let $\{T(\rho)\}_{0<\rho<\rho_{0}}$ be a family of circular crosscuts in $\Omega$ converging to $v$. For every $\rho \in\left(0, \rho_{0}\right)$ let $\theta(\rho)$ be the angle subtended by $T(\rho)$ at $v$. Then there exists $c \geqq 1$ such that

$$
\varphi_{1}(\omega) \leqq c \exp \left\{-\int_{\left|\omega-\nu^{\prime}\right|}^{\rho_{0}} \frac{\pi d \rho}{\theta(\rho) \rho}\right\} \quad(\omega \in U) .
$$

Proof. We shall need the following results:

Lemma 2.9. ([An, p. 280]) The Hardy inequality

$$
\int_{X} d(x)^{-2} f(x)^{2} d x \leqq c_{X} \int_{X}|\nabla f|^{2} d x \quad\left(f \in C_{c}^{\infty}(X)\right)
$$

holds for every simply connected bounded domain $X$ in $\mathbb{R}^{2}$. 
Lemma 2.10. ([B, p. 186]) Let $-\Delta$ be the Dirichlet Laplacian on a bounded John domain $X$ in $\mathbb{R}^{N}$, then $e^{\Delta t}$ is intrinsically ultracontractive. Moreover there exist $c_{X} \geqq 1$ and $\mu>0$ such that

$$
\left\|\varphi_{1}^{-1} e^{\Delta t} \varphi_{1}\right\|_{L^{1}\left(X, \varphi_{1}(x)^{2} d x\right) \rightarrow L^{\infty}\left(X, \varphi_{1}(x)^{2} d x\right)} \leqq c_{X} t^{-\mu} \quad(t>0) .
$$

Also, there exist $c \geqq 1$ and $\beta>0$ such that condition (iii) of Lemma 2.2 holds.

Let $\omega_{0}=g^{-1}(0)$. Then, by the two preceding lemmas, $-\Delta_{\Omega}$ satisfies the hypotheses of Lemma 2.2. Hence there exists $c \geqq 1$ such that

$$
\begin{aligned}
c^{-1} G_{\Omega}\left(\omega_{0}, \omega\right) & \leqq \varphi_{1}(\omega) \leqq c G_{\Omega}\left(\omega_{0}, \omega\right)=-c \ln |g(\omega)| \\
& =-c \ln (1-(1-|g(\omega)|)) \\
& \leqq 2 c(1-|g(\omega)|)
\end{aligned}
$$

for all $\omega \in \Omega$ with $|g(\omega)| \geqq \frac{1}{2}$. Since $U$ is a twisting cone of the type described in Proposition 2.7, $f\left(\left[t_{0}, 1\right)\right) \subseteq U$ for some $t_{0} \in(0,1)$ by (2.2). Hence for all $\rho \in$ $\left(0, \rho_{0}\right)$ there exists $\omega(\rho) \in V(\rho)$ such that $g(\omega(\rho)) \in(0,1)$. Thus, by Lemma 2.3,

$$
\lim _{\rho \downarrow 0}(1-g(\omega(\rho))) \exp \left\{\int_{\rho}^{\rho_{0}} \frac{\pi d \sigma}{\theta(\sigma) \sigma}\right\}<\infty
$$

exists. Hence (2.10) and (2.11) now give

$$
\varphi_{1}(\omega(\rho)) \leqq c \exp \left\{-\int_{\rho}^{\rho_{0}} \frac{\pi d \sigma}{\theta(\sigma) \sigma}\right\} \quad\left(0<\rho<\rho_{0}\right)
$$

for some $c \geqq 1$. Estimate (2.8) now follows from (2.12), (TC4), (TC6) and the elliptic Harnack inequality.

Theorem 2.11. Let $\tau_{n}$ be the constants given in Lemma 2.2. Then there exists $c \geqq 1$ such that

$$
\left|\nabla \varphi_{n}(\omega)\right| \leqq c E_{n} \tau_{n} \varphi_{1}(\omega) d(\omega)^{-1} \quad(\omega \in \Omega)
$$

for all $n=1,2,3, \ldots$. Futhermore, if there is a curve $\gamma:[0, \delta] \rightarrow \bar{\Omega}$ with $\gamma(0)=v$ and satisfying:

(i) $\gamma(t) \in U$ for all $t \in(0, \delta)$,

(ii) $\left|\gamma^{\prime}\right| \leqq M$ for some $M>0$,

(iii) $\gamma(t) \in V(t)$ for all $0<t<\delta<\rho_{0}$,

then we also have the lower bound

$$
\left|\nabla \varphi_{1}(\omega)\right| \geqq c^{-1} \varphi_{1}(\omega) d(\omega)^{-1} \quad(\omega \in U) .
$$

(Remark. If $\Omega$ is the snowflake domain, then a curve $\gamma:[0, \delta] \rightarrow \bar{\Omega}$ satisfying $\gamma(0)=v$ and (i) $\rightarrow$ (iii) in Theorem 2.11 exists for every $v \in \partial \Omega$.)

Proof. We shall assume that $D$ is on the $z$-plane where $z=x+i y$ and $\Omega$ is on the $\omega$-plane where $\omega=u+i v$. We let $g: \Omega \rightarrow D$ be a Riemann map with $g(v)=1$. Then

$$
\left|\frac{\partial(u, v)}{\partial(x, y)}\right|=\left|g^{\prime}(\omega)\right|^{-2}=\left|g^{\prime}\left(g^{-1}(z)\right)\right|^{-2} \quad(z \in D) .
$$


Let $T: L^{2}(\Omega) \rightarrow L^{2}\left(D,\left|g^{\prime}\left(g^{-1}(z)\right)\right|^{-2} d x d y\right)$ be the unitary operator defined by

$$
(T \psi)(z)=\psi\left(g^{-1}(z)\right) \quad\left(z \in D, \psi \in L^{2}(\Omega)\right) .
$$

Let $H \geqq 0$ be the self-adjoint operator in $L^{2}\left(D,\left|g^{\prime}\left(g^{-1}(z)\right)\right|^{-2} d x d y\right)$ unitarily equivalent to $-\Delta_{\Omega}$ in $L^{2}(\Omega)$ under $T$. We first note that the conformal invariance of the Green's function ([Ah, p. 249]) implies that if $G_{H}\left(z_{1}, z_{2}\right)$ is the Green's function of $H$, then

$$
G_{H}\left(z_{1}, z_{2}\right)=G_{\mathbb{D}}\left(z_{1}, z_{2}\right) \quad\left(z_{1}, z_{2} \in D\right)
$$

We have

$$
\varphi_{n}\left(\omega_{1}\right)=E_{n} \int_{\Omega} G_{\Omega}\left(\omega_{1}, \omega_{2}\right) \varphi_{n}\left(\omega_{2}\right) d u_{2} d v_{2},
$$

where $\omega_{\jmath}=u_{J}+i v_{j} \in \Omega, j=1,2$. Therefore

$$
\begin{gathered}
\frac{\partial \varphi_{n}}{\partial u_{1}}\left(\omega_{1}\right)=E_{n} \int_{\Omega} \frac{\partial G_{\Omega}}{\partial u_{1}}\left(\omega_{1}, \omega_{2}\right) \varphi_{n}\left(\omega_{2}\right) d u_{2} d v_{2} \\
=E_{n} \int_{\Omega}\left[\frac{\partial}{\partial u_{1}}\left(G_{\mathbb{D}}\left(g\left(\omega_{1}\right), g\left(\omega_{2}\right)\right)\right)\right] \varphi_{n}\left(\omega_{2}\right) d u_{2} d v_{2} \\
=E_{n} \int_{\Omega}\left(\frac{\partial G_{\mathbb{D}}}{\partial x_{1}} \frac{\partial x_{1}}{\partial u_{1}}+\frac{\partial G_{\mathbb{D}}}{\partial y_{1}} \frac{\partial y_{1}}{\partial u_{1}}\right) \varphi_{n}\left(\omega_{2}\right) d u_{2} d v_{2} \\
=E_{n}\left\{\int_{\Omega} \frac{\partial G_{\mathbb{D}}}{\partial x_{1}}\left(g\left(\omega_{1}\right), g\left(\omega_{2}\right)\right) \varphi_{n}\left(\omega_{2}\right) d u_{2} d v_{2} \frac{\partial x_{1}}{\partial u_{1}}\right. \\
\left.\quad+\int_{\Omega} \frac{\partial G_{\mathbb{D}}}{\partial y_{1}}\left(g\left(\omega_{1}\right), g\left(\omega_{2}\right)\right) \varphi_{n}\left(\omega_{2}\right) d u_{2} d v_{2} \frac{\partial y_{1}}{\partial u_{1}}\right\}
\end{gathered}
$$

Similarly we have

$$
\begin{aligned}
\frac{\partial \varphi_{n}}{\partial v_{1}}\left(\omega_{1}\right)=E_{n} & \left\{\int_{\Omega} \frac{\partial G_{\mathbb{D}}}{\partial x_{1}}\left(g\left(\omega_{1}\right), g\left(\omega_{2}\right)\right) \varphi_{n}\left(\omega_{2}\right) d u_{2} d v_{2} \frac{\partial x_{1}}{\partial v_{1}}\right. \\
& \left.+\int_{\Omega} \frac{\partial G_{\mathbb{D}}}{\partial y_{1}}\left(g\left(\omega_{1}\right), g\left(\omega_{2}\right)\right) \varphi_{n}\left(\omega_{2}\right) d u_{2} d v_{2} \frac{\partial y_{1}}{\partial v_{1}}\right\} .
\end{aligned}
$$

So writing

$$
\begin{aligned}
P_{n}\left(\omega_{1}\right) & =\int_{\Omega} \frac{\partial G_{\mathbb{D}}}{\partial x_{1}}\left(g\left(\omega_{1}\right), g\left(\omega_{2}\right)\right) \varphi_{n}\left(\omega_{2}\right) d u_{2} d v_{2} \\
& =\int_{\mathbb{D}} \frac{\partial G_{\mathbb{D}}}{\partial x_{1}}\left(g\left(\omega_{1}\right), z_{2}\right) \varphi_{n}\left(g^{-1}\left(z_{2}\right)\right)\left|g^{\prime}\left(g^{-1}\left(z_{2}\right)\right)\right|^{-2} d x_{2} d y_{2},
\end{aligned}
$$

and

$$
Q_{n}\left(\omega_{1}\right)=\int_{\mathbb{D}} \frac{\partial G_{\mathbb{D}}}{\partial y_{1}}\left(g\left(\omega_{1}\right), z_{2}\right) \varphi_{n}\left(g^{-1}\left(z_{2}\right)\right)\left|g^{\prime}\left(g^{-1}\left(z_{2}\right)\right)\right|^{-2} d x_{2} d y_{2}
$$


we have

$$
\begin{aligned}
\left|\nabla \varphi_{n}\left(\omega_{1}\right)\right|^{2}= & E_{n}^{2}\left\{\left[P\left(\omega_{1}\right) \frac{\partial x_{1}}{\partial u_{1}}+Q\left(\omega_{1}\right) \frac{\partial y_{1}}{\partial u_{1}}\right]^{2}\right. \\
& \left.+\left[P\left(\omega_{1}\right) \frac{\partial x_{1}}{\partial v_{1}}+Q\left(\omega_{1}\right) \frac{\partial y_{1}}{\partial v_{1}}\right]^{2}\right\} \\
= & E_{n}^{2}\left\{P\left(\omega_{1}\right)^{2}\left[\left(\frac{\partial x_{1}}{\partial u_{1}}\right)^{2}+\left(\frac{\partial x_{1}}{\partial v_{1}}\right)^{2}\right]\right. \\
& +Q\left(\omega_{1}\right)\left[\left(\frac{\partial y_{1}}{\partial u_{2}}\right)^{2}+\left(\frac{\partial y_{1}}{\partial v_{1}}\right)^{2}\right] \\
& \left.+2 P\left(\omega_{1}\right) Q\left(\omega_{1}\right)\left[\left(\frac{\partial x_{1}}{\partial v_{1}}\right)\left(\frac{\partial y_{1}}{\partial v_{1}}\right)+\left(\frac{\partial x_{1}}{\partial u_{1}}\right)\left(\frac{\partial y_{1}}{\partial u_{1}}\right)\right]\right\} \\
= & E_{n}^{2}\left\{P\left(\omega_{1}\right)^{2}\left[\left(\frac{\partial x_{1}}{\partial u_{1}}\right)^{2}+\left(\frac{\partial y_{1}}{\partial u_{1}}\right)^{2}\right]\right. \\
& \left.+Q\left(\omega_{1}\right)^{2}\left[\left(\frac{\partial x_{1}}{\partial v_{1}}\right)^{2}+\left(\frac{\partial y_{1}}{\partial v_{1}}\right)^{2}\right]\right\} \\
= & E_{n}^{2}\left\{P\left(\omega_{1}\right)^{2}+Q\left(\omega_{1}\right)^{2}\right\}\left|g^{\prime}\left(\omega_{1}\right)\right|^{2} .
\end{aligned}
$$

Now (see [HK, p. 26])

$$
G_{\mathbb{D}}\left(z_{1}, z_{2}\right)= \begin{cases}\ln \left(\frac{\left.\left|z_{1}-\right| z_{2}\right|^{-2} z_{2}|| z_{2} \mid}{\left|z_{1}-z_{2}\right|}\right) & \left(z_{2} \neq 0\right) \\ \ln \left(\left|z_{1}\right|^{-1}\right) & \left(z_{2}=0\right) .\end{cases}
$$

So if $z_{2} \neq 0$, then

$$
\begin{aligned}
G_{\mathbb{D}}\left(z_{1}, z_{2}\right)=\frac{1}{2}\left\{\ln \left[\left(x_{1}^{2}+y_{1}^{2}\right)\left(x_{2}^{2}+y_{2}^{2}\right)-2 x_{1} x_{2}-2 y_{1} y_{2}+1\right]\right. \\
\left.\quad-\ln \left[\left(x_{1}^{2}+y_{1}^{2}\right)+\left(x_{2}^{2}+y_{2}^{2}\right)-2 x_{1} x_{2}-2 y_{1} y_{2}\right]\right\} .
\end{aligned}
$$

Thus if $z_{2} \neq 0$, then

$$
\begin{aligned}
\frac{\partial G_{\mathbb{D}}}{\partial x_{1}}= & \frac{x_{1}\left(x_{2}^{2}+y_{2}^{2}\right)-x_{2}}{\left(x_{1}^{2}+y_{1}^{2}\right)\left(x_{2}^{2}+y_{2}^{2}\right)-2 x_{1} x_{2}-2 y_{1} y_{2}+1} \\
& -\frac{x_{1}-x_{2}}{\left(x_{1}^{2}+x_{2}^{2}\right)+\left(y_{1}^{2}+y_{2}^{2}\right)-2 x_{1} x_{2}-2 y_{1} y_{2}} .
\end{aligned}
$$

Hence, for some $c \geqq 1$, we have

$$
\begin{aligned}
\left|\frac{\partial G_{\mathbb{D}}}{\partial x_{l}}\right| & =\frac{\left.\left|z_{2}\right|\left|x_{1}\right| z_{2}\left|-x_{2}\right| z_{2}\right|^{-1} \mid}{\left.\left.\left|z_{1}\right| z_{2}\left|-z_{2}\right| z_{2}\right|^{-1}\right|^{2}} \quad\left(z_{1} \neq z_{2}, z_{2} \neq 0\right) \\
& \leqq\left.\left.\left|z_{1}\right| z_{2}\left|-z_{2}\right| z_{2}\right|^{-1}\right|^{-1} \\
& \leqq c\left|z_{1}-z_{2}\right|^{-1}\left|z_{2}\right|^{-1} .
\end{aligned}
$$

Since $\Omega$ is a bounded John domain, there exist $c \geqq 1$ and $\beta>0$ such that

$$
c^{-1} d(\omega)^{\beta} \leqq \varphi_{1}(\omega) \quad(\omega \in \Omega) .
$$


Thus Lemmas 2.2 and 2.6 together with (2.10) and (2.17) imply that, for some $c \geqq 1$,

$$
\begin{aligned}
\left|\varphi_{n}\left(g^{-1}(z)\right)\right|\left|g^{\prime}\left(g^{-1}(z)\right)\right|^{-2} & \leqq c \tau_{n} \varphi_{1}\left(g^{-1}(z)\right)(1-|z|)^{-2} d\left(g^{-1}(z)\right)^{2} \\
& \leqq c \tau_{n}(1-|z|)^{-1} \varphi_{1}\left(g^{-1}(z)\right)^{2 / \beta} \\
& \leqq c \tau_{n}(1-|z|)^{-1+2 / \beta}
\end{aligned}
$$

for all $z \in D$. Then inequalities (2.16) and (2.18) now imply that $P$ is a continuous function on $\bar{\Omega}$. (To see this we only need to show that for all $\delta \in(0,1)$, we have

$$
I_{\partial}=\int_{x_{2}=-A}^{A} \int_{x_{1}=0}^{1} x_{1}^{-\delta}\left(x_{1}^{2}+x_{2}^{2}\right)^{-\frac{1}{2}} d x_{1} d x_{2}<\infty .
$$

But

$$
\begin{aligned}
I_{\delta} & =\int_{-A}^{A}\left(\int_{0}^{\tan ^{-1}\left(x_{2}^{-1}\right)}\left(x_{2} \sec u\right)^{-1}\left(x_{2}^{-\delta} \tan ^{-\delta} u \cdot x_{2} \sec ^{2} u d u\right)\right) d x_{2} \\
& =\left(\int_{-A}^{A} x_{2}^{-\delta} d x_{2}\right)\left(\int_{0}^{\pi / 2} \tan ^{-\delta} u \sec u d u\right) \\
& <\infty .)
\end{aligned}
$$

Similarly we can show that $Q$ is a continuous function on $\bar{\Omega}$. Inequalities (2.15), (2.16) and (2.18) now imply that

$$
\left|\nabla \varphi_{n}\left(\omega_{1}\right)\right|^{2} \leqq c E_{n}^{2} \tau_{n}^{2}\left|g^{\prime}\left(\omega_{1}\right)\right|^{2} \quad\left(\omega_{1} \in \Omega\right) ;
$$

hence (2.6) and (2.10) give

$$
\begin{aligned}
\left|\nabla \varphi_{n}\left(\omega_{1}\right)\right|^{2} & \leqq c E_{n}^{2} \tau_{n}^{2}\left(1-\left|g\left(\omega_{1}\right)\right|\right)^{2} d\left(\omega_{1}\right)^{-2} \quad\left(\omega_{1} \in \Omega\right) \\
& \leqq c E_{n}^{2} \tau_{n}^{2} \varphi_{1}\left(\omega_{1}\right)^{2} d\left(\omega_{1}\right)^{-2}
\end{aligned}
$$

which implies (2.13). To obtain a lower bound for $\left|\nabla \varphi_{1}\right|$ we let $\gamma:[0, \delta] \rightarrow \bar{\Omega}$ with $\gamma(0)=v$ and satisfying conditions $(\mathrm{i}) \rightarrow($ iii $)$. We now show that

$$
P_{1}(v)^{2}+Q_{1}(v)^{2}>0
$$

Suppose the contrary that

$$
L(\omega)=\left[P_{1}(\omega)^{2}+Q_{1}(\omega)^{2}\right]^{\frac{1}{2}} \text { as } \omega \rightarrow v .
$$

Put

$$
\psi(t)=\varphi_{1}(\gamma(t)) \quad(0 \leqq t \leqq \delta) .
$$

Then (2.10), (2.15) and Lemma 2.6 imply that

$$
\begin{aligned}
\left|\psi^{\prime}(t)\right| & \leqq\left|\nabla \varphi_{1}(\gamma(t))\right|\left|\gamma^{\prime}(t)\right| \quad(0 \leqq t \leqq \delta) \\
& =E_{1} L(\gamma(t))\left|g^{\prime}(\gamma(t))\right|\left|\gamma^{\prime}(t)\right| \\
& \leqq 2 E_{1} M L(\gamma(t))(1-|g(\gamma(t))|) d(\gamma(t))^{-1} \\
& \leqq c L(\gamma(t)) \varphi_{1}(\gamma(t)) d(\gamma(t))^{-1}
\end{aligned}
$$


Hence (iii) implies that

$$
\psi^{\prime}(t) / \psi(t) \leqq c L(\gamma(t)) t^{-1} \quad(0 \leqq t \leqq \delta) .
$$

Integrating (2.21) we obtain

$$
-\ln \varphi_{1}(\gamma(t)) \leqq c+c \int_{t}^{\delta} L(\gamma(\tau)) \tau^{-1} d \tau .
$$

By (2.20) given any $\varepsilon>0$, there exists a sufficiently small $r_{0}(\varepsilon)>0$ such that

$$
L(\gamma(t)) \leqq \varepsilon \quad \text { if } \quad 0 \leqq t \leqq r_{0}(\varepsilon)
$$

So, for some $k_{1}$, with $k_{1} \geqq 1$, we have

$$
\begin{aligned}
-\ln \varphi_{1}(\gamma(t)) & \leqq k_{1}+\int_{t}^{r_{0}(\varepsilon)} L(\gamma(\tau)) \tau^{-1} d \tau \quad\left(0 \leqq t \leqq r_{0}\right) \\
& \leqq k_{1}+\varepsilon \int_{t}^{r_{0}(\varepsilon)} \tau^{-1} d \tau \\
& =k_{1}-\varepsilon \ln t .
\end{aligned}
$$

Hence, for some $k_{2} \geqq 1$, we have

$$
\varphi_{1}(\gamma(t)) \geqq k_{2} t^{i} \quad\left(0 \leqq t \leqq r_{0}(\varepsilon)\right)
$$

which, since $\varepsilon>0$ was arbitrarily small, contradicts Proposition 2.12 below. Thus (2.19) holds. Estimate (2.14) now follows from (2.15), (2.19), (2.16) and (2.10).

Proposition 2.12. There exists $c \geqq 1$ such that

$$
\varphi_{1}(\omega) \leqq c d(\omega)^{\frac{1}{2}} \quad(\omega \in \Omega) .
$$

(Clearly, as the example of the cardiord shows, the exponent $\frac{1}{2}$ on the right is sharp among the class of John domains.)

Proof. By (2.5) and (2.6) we have

$$
1-|g(\omega)| \leqq c d(\omega)^{\frac{1}{2}} \quad(\omega \in \Omega)
$$

for some $c \geqq 1$. Hence (2.10) gives

$$
\varphi_{1}(\omega) \leqq c(1-|g(\omega)|) \leqq c d(\omega)^{\frac{1}{2}} \quad(\omega \in \Omega)
$$

Corollary 2.13. Let $\mu>0$ be chosen so that (2.9) holds. Then there exists $c \geqq 1$ such that

$$
\left|\nabla_{\omega_{2}} K\left(t, \omega_{1}, \omega_{2}\right)\right| \leqq c m(t) \varphi_{1}\left(\omega_{1}\right) \varphi_{1}\left(\omega_{2}\right) d\left(\omega_{2}\right)^{-1} \quad(\omega \in \Omega),
$$


where $K\left(t, \omega_{1}, \omega_{2}\right)$ is the heat kernel of $e^{\Delta t}$ and

$$
m(t)=\sum_{n=1}^{\infty} E_{n}^{2 \mu+1} e^{-E_{n} t}<\infty \quad(t>0) .
$$

Proof. Let $\tau_{n}$ be the constants given in Lemma 2.2. Then (2.9) gives

$$
\tau_{n}=\inf \left\{c t^{-\mu} e^{\left(E_{n}-E_{1}\right) t}: t>0\right\} \leqq E_{n}^{\mu}
$$

Thus by (2.13) and Lemma 2.2 we have

$$
\begin{aligned}
\left|\nabla_{\omega_{2}} K\left(t, \omega_{1}, \omega_{2}\right)\right| & \leqq \sum_{n=1}^{\infty} e^{-E_{n} t}\left|\varphi_{n}\left(\omega_{1}\right)\right|\left|\nabla \varphi_{n}\left(\omega_{2}\right)\right| \\
& \leqq c \sum_{n=1}^{\infty} e^{-E_{n} t} E_{n}^{\mu} \varphi_{1}\left(\omega_{1}\right) E_{n}^{\mu+1} \varphi_{1}\left(\omega_{2}\right) d\left(\omega_{2}\right)^{-1}
\end{aligned}
$$

\section{Snowflake Domain}

In this section we apply the results in Sect. 2 to the special case where $\Omega$ is the domain bounded by the Koch snowflake curve (see [F, Fig. 0.2(b), p. XV]). It will be clear that our method also applies to other domains with self-similar (fractal) boundaries, such as the square snowflake considered in [S, SGM, SG].

Notations. We let $\{P(n)\}_{n=1}^{\infty}$ be the usual sequence of interior polygonal approximations of $\Omega$ (i.e., $P(n)$ is obtained from $P(n-1)$ by adding a "middle-third" triangle on every segment of $\partial P(n-1))$. For every $n$ we let $A c(n)$ be the set of all acute angle vertices of $P(n)$ and let $O b(n)$ be the set of all obtuse angle vertices of $P(n)$. (All angles of polygons are measured from the inside.) If $s_{j}\left(j=1,2, \ldots, 3 \cdot 4^{n-1}\right)$ are the segments making up $\partial P(n)$ and if for each $j$ we let $C a\left(s_{\jmath}\right)$ be the (ternary) Cantor set of $s_{J}$, then we define $C a(n)=\bigcup_{J=1}^{3 \cdot 4^{n-1}} C a\left(s_{\jmath}\right)$. We also define the subsets $A c, O b$ and $\mathrm{Ca}$ of $\partial \Omega$ by:

$$
A c=\bigcup_{n=1}^{\infty} A c(n), O b=\bigcup_{n=1}^{\infty} O b(n), C a=\left\{\bigcup_{n=1}^{\infty} C a(n)\right\} \backslash(A c \cup O b) .
$$

The position of each point $v$ in $\partial \Omega \backslash(A c \cup O b \cup C a)$ can be specified using an infinite sequence $q_{1}, q_{2}, q_{3}, \ldots$, where

$$
\begin{aligned}
& q_{1} \in\{1,2,3,4,5,6\} \\
& q_{n} \in\{1,2,3,4,5\} \text { for } n \geqq 2
\end{aligned}
$$

(see Fig. 2 below). For the rest of this section $U$ will again denote a twisting cone in $\Omega$ with vertex at $v \in \partial \Omega$ of the type described in Proposition 2.7.

Proposition 3.1. (i) If $v \in A c$, then

$$
\varphi_{1}(\omega) \leqq c|\omega-v|^{\pi ;} \quad(\omega \in U)
$$

for some $c \geqq 1$ and $\gamma \in\left(\frac{3}{2 \pi}, \frac{3}{\pi}\right)$.

(ii) If $v \in \overline{O b}$, then

$$
\varphi_{1}(\omega) \leqq c|\omega-v|^{\pi i} \quad(\omega \in U)
$$

for some $c \geqq 1$ and $\gamma \in\left(\frac{3}{5 \pi}, \frac{3}{4 \pi}\right)$. 


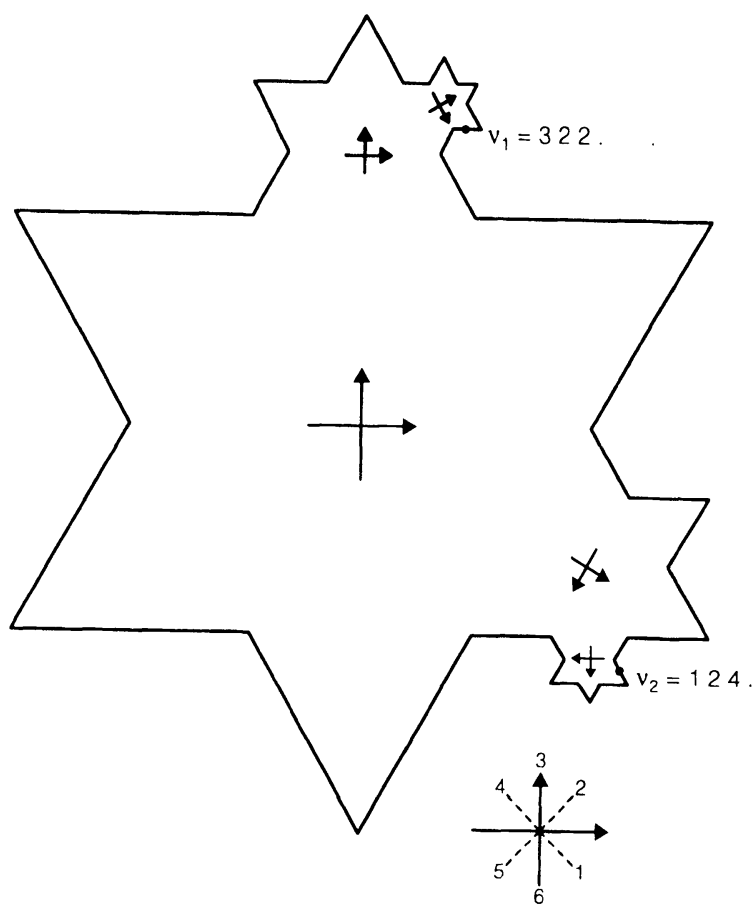

Fig. 2. Labelling of the boundary points of the snowflake domain

(iii) If $v \in C a$, then there exists a function $\alpha:\left(0, \rho_{0}\right) \rightarrow(0, \infty)$ such that

$$
\alpha(\rho) \uparrow \infty \quad \text { as } \quad \rho \downarrow 0
$$

and

$$
|(1)-v| \alpha(|\omega-v|) \leqq \varphi_{1}(\omega) \quad(\omega \in U)
$$

Remark. The values of $\gamma$ in (i) and (ii) are independent of $v \in A c$ or $v \in O b$. They are given by (3.2) below and can be regarded as the reciprocal of the average angle of $\partial \Omega$ at $v$.

Proof. We first prove (i) and (ii). Let $\{T(\rho)\}_{0<\rho<\rho_{0}}$ and $\theta(\rho)$ be as in Theorem 2.8 and let

$$
\tilde{\theta}(r)=\theta\left(e^{-r}\right) \quad\left(r>-\ln \rho_{0}\right) .
$$

By the self-similarity of $\partial \Omega$ near $v$ we see that

$$
\tilde{\theta}(r)=\tilde{\theta}(r+\ln 3) \quad\left(r>-\ln \rho_{o}\right) .
$$


Hence

$$
\begin{aligned}
\int_{\rho}^{\rho_{0}} \frac{d \sigma}{\theta(\sigma) \sigma}= & \int_{-\ln \rho_{0}}^{-\ln \rho} \tilde{\theta}(r)^{-1} d r \\
= & {\left[(\ln 3)^{-1}\left(\ln \rho_{0}-\ln \rho\right)-\eta(\rho)\right] \int_{k}^{k+\ln 3} \tilde{\theta}(r)^{-1} d r } \\
& +\int_{-\ln \rho+\ln \rho_{0}-\eta(\rho) \ln 3}^{-\ln \rho} \tilde{\theta}(r)^{-1} d r \\
= & \left(\ln \rho_{0}-\ln \rho\right)(\ln 3)^{-1} \int_{h}^{k+\ln 3} \tilde{\theta}(r)^{-1} d r+0(1),
\end{aligned}
$$

where

$$
0 \leqq \eta(\rho)<1 \quad\left(\rho \in\left(0, \rho_{0}\right)\right)
$$

Hence, for some $c \geqq 1$, we have

$$
c^{-1} \rho^{i \pi} \leqq \exp \left\{-\int_{\rho}^{\rho_{0}} \frac{\pi d \sigma}{\theta(\sigma) \sigma}\right\} \leqq c \rho^{i \pi} \quad\left(0<\rho<\rho_{0}\right),
$$

where

$$
\gamma=(\ln 3)^{-1} \int_{k}^{k+\ln 3} \theta\left(e^{-t}\right)^{-1} d r
$$

(i) and (ii) now follow from (3.1), (3.2) and Theorem 2.8. To prove (iii) we let $v \in C a$ and let $q_{1} q_{2} q_{3} \cdots, q_{l}=0$ or 2 , be the ternary expansion of $v$ with respect to a segment $s$ of $\partial P(n)$. We assume that $\rho_{0}$ is sufficiently small and to each $\rho \in\left(0, \rho_{0}\right)$ we associate two numbers $\gamma_{+}(\rho), \gamma_{-}(\rho) \in[0, \pi / 6)$ as illustrated in Fig. 3 .

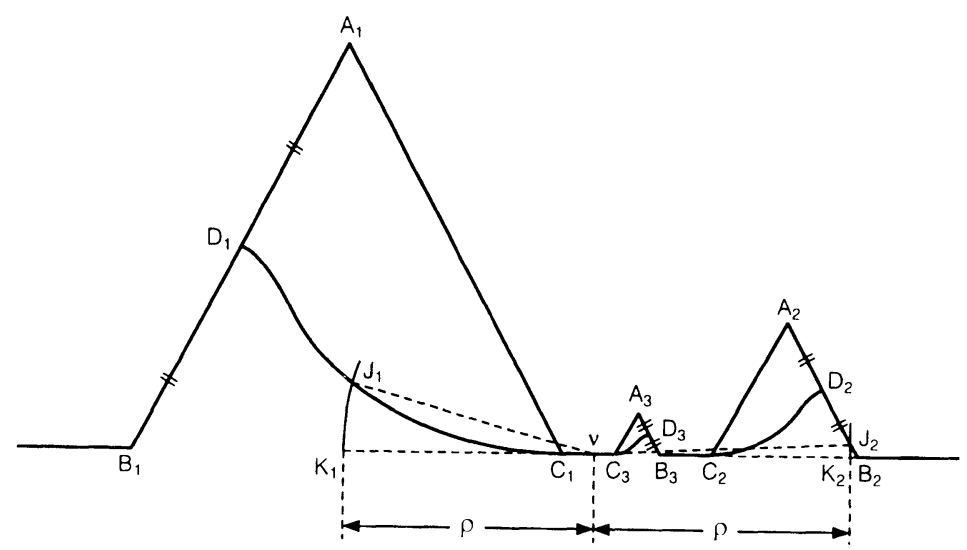

Fig. 3. (1) $\triangle A_{1} B_{1} C_{2}$ and the curve $C_{1} D_{1}$ can be obtained from $\triangle A_{2} B_{2} C_{2}$ and the curve $C_{2} D_{2}$ or from $\triangle A_{3} B_{3} C_{3}$ and the curve $C_{3} D_{3}$ by a reflection, translation and dilation. (2) The curve $D_{1} C_{1} v$ is $C^{\infty}$. (3) $\gamma_{-}(\rho)$ and $\gamma_{+}(\rho)$ are the size of $\varangle J_{1} v K_{1}$ and $\varangle J_{2} v K_{2}$, respectively, in radians 
Then one can check that $\gamma_{+}$and $\gamma_{-}$are locally absolutely continuous functions on $\left(0, \rho_{0}\right)$ and that there exists $c \geqq 1$ such that

$$
\left|\gamma_{ \pm}^{\prime}(\rho)\right| \leqq c \rho^{-1} \quad\left(0<\rho<\rho_{0}\right)
$$

Let

$$
\theta_{+}(\rho)=\gamma_{+}(\rho) /(-\ln \rho) \quad\left(0<\rho<\rho_{0}\right)
$$

and let

$$
\theta_{-}(\rho)=-\pi-\gamma_{-}(\rho) /(-\ln \rho) \quad\left(0<\rho<\rho_{0}\right) .
$$

Let $\Omega^{\prime}$ be the subregion of $\Omega$ defined by

$$
\Omega^{\prime}=\left\{v+\rho e^{l)}: 0<\rho<\rho_{0} \text { and } \theta_{-}(\rho)<\theta<\theta_{+}(\rho)\right\} .
$$

We now show that

$$
\int_{0}^{\rho_{0}}\left[\theta_{+}(\rho)-\theta_{-}(\rho)\right]^{-1}\left\{\theta_{+}^{\prime}(\rho)^{2}+\theta_{-}^{\prime}(\rho)^{2}\right\} \rho d \rho<\infty,
$$

so that we can apply Lemma 2.3. We have

$$
\begin{aligned}
\rho \theta_{ \pm}^{\prime}(\rho)^{2}= & {\left[(-\ln \rho)^{-1} \gamma_{ \pm}^{\prime}(\rho)+\gamma_{ \pm}(\rho)(-\ln \rho)^{-2} \rho^{-1}\right]^{2} \rho } \\
= & {\left[(-\ln \rho)^{2} \gamma_{ \pm}^{\prime}(\rho)^{2}+2 \rho^{-1}(-\ln \rho)^{-3} \gamma_{ \pm}^{\prime}(\rho) \gamma_{ \pm}(\rho)\right.} \\
& \left.+\gamma_{ \pm}(\rho)^{2}(-\ln \rho)^{-4} \rho^{-2}\right] \rho .
\end{aligned}
$$

Thus (3.3) implies that

$$
\rho \theta_{ \pm}^{\prime}(\rho)^{2} \leqq c \rho^{-1}(-\ln \rho)^{-2} \quad\left(0<\rho<\rho_{0}\right)
$$

for some $c \geqq 1$. Hence

$$
\begin{aligned}
& \int_{0}^{\rho_{0}}\left[\theta_{+}(\rho)-\theta_{-}(\rho)\right]^{-1}\left\{\theta_{+}^{\prime}(\rho)^{2}+\theta_{-}^{\prime}(\rho)^{2}\right\} \rho d \rho \\
& \quad \leqq \int_{0}^{\rho_{0}} 2 \pi^{-1} c \rho^{-1}(-\ln \rho)^{-2} d \rho \\
& \quad=2 \pi^{-1} c \int_{-\ln \rho_{0}}^{\infty} r^{-2} d r<\infty .
\end{aligned}
$$

Let $\varphi_{\Omega^{\prime}}>0$ be the ground state eigenfunction of $-\Delta_{\Omega^{\prime}}$ normalized by $\left\|\varphi_{\Omega^{\prime}}\right\|_{2}=1$. Let $U_{\Omega^{\prime}}$ be a twisting cone inside $\Omega^{\prime}$ with vertex at $v$ of the type described in Proposition 2.7. Then a proof similar to that of Theorem 2.8 implies that, for some $c \geqq 1$,

$$
\begin{array}{r}
c^{-1} \exp \left\{-\int_{\left|(\omega)-\nu^{\prime}\right|}^{\rho_{0}} \frac{\pi d \tau}{\left[\theta_{+}(\tau)-\theta_{-}(\tau)\right] \tau}\right\} \leqq \varphi_{\Omega^{\prime}}(\omega) \\
\leqq c \exp \left\{-\int_{\mid\left(\nu-\nu^{\prime} \mid\right.}^{\rho_{0}} \frac{\pi d \tau}{\left[\theta_{+}(\tau)-\theta_{-}(\tau)\right] \tau}\right\}
\end{array}
$$

for all $\omega \in U_{\Omega^{\prime}}$. Writing

$$
\gamma(\rho)=\gamma_{+}(\rho)+\gamma_{-}(\rho) \quad\left(0<\rho<\rho_{0}\right)
$$


we have

$$
\begin{aligned}
\int_{\rho}^{\rho_{0}} \frac{\pi d \tau}{\left[\theta_{+}(\tau)-\theta_{-}(\tau)\right] \tau} & =\int_{\rho}^{\rho_{0}} \frac{\pi d \tau}{\left[\pi+\gamma(\tau)(-\ln \tau)^{-1}\right] \tau} \quad\left(0<\rho<\rho_{0}\right) \\
& =\int_{\rho}^{\rho_{0}}\left[1-\frac{\gamma(\tau)}{\pi(-\ln \tau)+\gamma(\tau)}\right] \frac{d \tau}{\tau} \\
& =\left(\ln \rho_{0}-\ln \rho\right)-\int_{\rho}^{\rho_{0}} \frac{\gamma(\tau)}{\pi(-\ln \tau)+\gamma(\tau)} \cdot \frac{d \tau}{\tau}
\end{aligned}
$$

Since $0 \leqq \gamma(\tau) \leqq \pi / 3$, there exists $c \geqq 1$ with

$$
c^{-1} \tau^{-1}(-\ln \tau)^{-1} \leqq \tau^{-1}[\pi(-\ln \tau)+\gamma(\tau)]^{-1} \leqq c \tau^{-1}(-\ln \tau)^{-1}
$$

for all $\tau \in\left(0, \rho_{0}\right)$. Let

$$
\Gamma(r)=\gamma\left(e^{-r}\right) \quad\left(r>-\ln \rho_{0}\right) .
$$

Then, by the definition of the functions $\gamma_{ \pm}$, one can check that there exist $c_{3}, c_{4} \geqq 1$ with the following properties:

(a) If $r>-\ln \rho_{0}$ and if $\Gamma(r) \geqq c_{3}^{-1}$, then there exists an interval $\left(\sigma_{1}, \sigma_{2}\right) \subseteq$ $\left(-\ln \rho_{0}, \infty\right)$ of length at least $c_{4}^{-1}$ such that $r \in\left(\sigma_{1}, \sigma_{2}\right)$ and

$$
\Gamma(\tau) \geqq c_{3}^{-1} \quad\left(\tau \in\left(\sigma_{1}, \sigma_{2}\right)\right) .
$$

(b) If $r>-\ln \rho_{0}$ and if $\Gamma(r)<c_{3}^{-1}$, then there does not exist any interval $\left(\sigma_{1}, \sigma_{2}\right) \subseteq\left(-\ln \rho_{0}, \infty\right)$ of length greater than $2 \ln 2$ such that $r \in\left(\sigma_{1}, \sigma_{2}\right)$ and

$$
\Gamma(\tau)<c_{3}^{-1} \quad\left(\tau \in\left(\sigma_{1}, \sigma_{2}\right)\right) .
$$

Hence we have

$$
\begin{aligned}
\int_{\rho}^{\rho_{0}} \frac{\gamma(\tau)}{[\pi(-\ln \tau)+\gamma(\tau)]} \cdot \frac{d \tau}{\tau} & \geqq c^{-1} \int_{\rho}^{\rho_{0}} \frac{\gamma(\tau) d \tau}{(-\ln \tau) \tau} \\
& =c^{-1} \int_{-\ln \rho_{0}}^{-\ln \rho} \frac{\Gamma(r)}{r} d r \\
& \rightarrow \infty \text { as } \rho \downarrow 0
\end{aligned}
$$

Equations (3.4), (3.5) and (3.6) now imply that

$$
|\omega-v| \tilde{\alpha}(|\omega-v|) \leqq \varphi_{\Omega^{\prime}}(\omega) \quad\left(\omega \in U_{\Omega^{\prime}}\right)
$$

where $\tilde{\alpha}:\left(0, \rho_{0}\right) \rightarrow(0, \infty)$ is a function such that

$$
\tilde{x}(\rho) \uparrow \infty \quad \text { as } \quad \rho \downarrow 0 .
$$

Thus there exists $c \geqq 1$ such that

$$
c^{-1}|\omega-v| \tilde{\alpha}(|\omega-v|) \leqq \varphi_{1}(\omega) \quad\left(\omega \in U_{\Omega^{\prime}}\right) .
$$

The elliptic Harnack inequality and the properties of twisting cones now enable (3.7) to be extended to all $\omega \in U$ (with a different $c \geqq 1$ ), thus proving (iii). 
Theorem 3.2. (i) If $v \in A c$, then

$$
\left|\nabla \varphi_{1}(\omega)\right| \rightarrow 0 \quad \text { as } \quad \omega \rightarrow v \quad \text { with } \quad \omega \in U .
$$

(ii) If $v \in O b \cup C a$, then

$$
\left|\nabla \varphi_{1}(\omega)\right| \rightarrow \infty \quad \text { as } \quad \omega \rightarrow v \text { with } \omega \in U .
$$

(iii) If $y$ belongs to the (ternary) Cantor set of the line segment joining two adjacent acute angle vertices of $P(n)$, separated by only one obtuse angle vertex, for any $n \in \mathbb{N}$, then

$$
\left|\nabla \varphi_{1}(\omega)\right| \rightarrow 0 \quad \text { as } \quad \omega \rightarrow v \text { with } \omega \in U .
$$

Proof. (i) is a consequence of Proposition 3.1 (i) and (2.13). If $v \in C a$, then the result follows from Proposition 3.1 (iii) and (2.14). If $v \in O b$, then, by considering a finite wedge at $v$,

$$
c^{-1}|\omega-v|^{3 / 4} \leqq \varphi_{1}(\omega) \quad(\omega \in U)
$$

for some $c \geqq 1$. Therefore the result for $v \in O b$ follows from (3.8) and (2.14). Finally suppose $v$ is as in (iii) and let $\{T(\rho)\}_{0<\rho<\rho_{0}}$ and $\theta(\rho)$ be as in Theorem 2.8. Then $\theta(\rho)<\pi$ for almost all $\rho$ in $\left(0, \rho_{0}\right)$. Hence the result follows from (2.8) and (2.13).

Remarks. (i) With more effort one can use Lemma 2.3, Theorems 2.8 and 2.11 to determine whether $\left|\nabla \varphi_{1}(\omega)\right|$ tends to 0 or $\infty$ as $\omega \rightarrow v$ with $\omega \in U$ for $v \in \partial \Omega \backslash(A c \cup O b \cup C a)$ other than those described in (iii). For example, one can show that if $v \in \partial \Omega \backslash(A c \cup O b \cup C a)$ and if the sequence representing $v$ eventually becomes

$$
3323332333323333323333332 \cdots \text {, }
$$

then

$$
\left|\nabla \varphi_{1}(\omega)\right| \rightarrow 0 \text { as } \omega \rightarrow v \text { with } \omega \in U .
$$

(ii) By [O, Theorem 2] the set of boundary points $v$ considered in Theorem 3.2 has zero harmonic measure. A detailed description of the behaviour of $\left|\nabla \varphi_{1}\right|$ near generic boundary points (with respect to harmonic measure) remains open.

Acknowledgement. We wish to thank Bernard Sapoval, Robert S. Strichartz and Rodrigo Banuelos for very helpful discussions and comments. The second author also thanks the Mathematics Department, University of California at Riverside, where part of this work was carried out.

\section{References}

[Ah] Ahlfors, L.V.: Complex analysis. 2nd edition, New York: McGraw-Hill, 1966

[An] Ancona, A.: On strong barriers and an inequality of Hardy for domains in $\mathbb{R}^{N}$. J. London Math. Soc. 34, 274-290 (1986)

[B] Banuelos, R.: Intrinsic ultracontractivity and eigenfunction estimates for Schrödinger operators. J. Funct. Anal. 100, 181-206 (1991)

[D] Davies, E.B.: Heat kernels and spectral theory. Cambridge: Cambridge Univ. Press, 1989 
[DS] Davies, E.B., Simon, B.: Untracontractivity and heat kernels for Schrödinger operators and Dirichlet Laplacians. J. Funct. Anal. 59, 335-395 (1984)

[F] Falconer, K.J.: Fractal geometry. Wiley, Chichester, 1990

[HK] Hayman, W.K., Kennedy, P.B.: Subharmonic functions. vol. 1, London: Academic Press, 1976

[L] Lapidus, M.L.: Vibrations of fractal drums, the Riemann hypothesis, waves in fractal media, and the Weyl-Berry conjecture. In: "Ordinary and Partial Differential Equations", vol. IV B.D. Sleeman, R.J. Jarvis, (eds.) Pitman Research Notes in Math. Series 289, London: Longman, 1993 pp. 126-209

[O] Oksendal, B.: Brownian motion and sets of harmonic measure zero. Pacific J. Math. 95, 193-204 (1981)

[P] Pommerenke, Ch.: Boundary behaviour of conformal maps, Springer, 1992

[RW] Rodin, B., Warschawski, S.E.: Estimates for conformal maps of strip domains without boundary regularity. Proc. London Math. Soc. (3) 99, 356-384 (1979)

[S] Sapoval, B.: Experimental observation of local modes in fractal drums. Physica D 38, 296-298 (1989)

[SGM] Sapoval, B., Gobron, Th., Margolina, A.: Vibrations of fractal drums. Phys. Rev. Lett. 67, 2974-2977 (1991)

[SG] Sapoval, B., Gobron, Th.: Vibrations of strongly irregular or fractal resonators. Phys. Rev. E 47, no. 5, 3013-3024 (1993)

Communicated by B. Simon 